Open Access Full Text Article

\title{
Large-Scale Analysis Reveals the Specific Clinical and Immune Features of DGCR5 in Glioma
}

This article was published in the following Dove Press journal:

OncoTargets and Therapy

\section{Xuechao Wu* \\ Peng Hou* \\ Yun Qiu \\ Qing Wang \\ Xiaojie Lu}

Department of Neurosurgery, The Affiliated Wuxi No.2 People's Hospital of Nanjing Medical University, Wuxi,

People's Republic of China

*These authors contributed equally to this work
Correspondence: Xiaojie Lu

Department of Neurosurgery, The

Affiliated Wuxi, No.2 People's Hospital of

Nanjing Medical University, Wuxi,

People's Republic of China

Email luxiaojiewuxi@I63.com
Purpose: Long non-coding RNA DGCR5 plays different roles in different types of cancer. The purpose of this study was to investigate the clinicopathological features, potential biological functions and prognostic significance of DGCR5 in glioma in a large-scale study. Materials and Methods: A total of 697 RNA-seq data from The Cancer Genome Atlas (TCGA) and 301 mRNA microarray data from Chinese Glioma Genome Atlas (CGGA) were enrolled in this study. R language was used as the main tool for statistical analysis and graphical work.

Results: DGCR5 showed a negative correlation with the WHO grade of malignancy in glioma. Specifically, DGCR5 expression was significantly decreased in GBM and IDH wildtype glioma. Gene ontology analysis showed that DGCR5 was predominantly enriched in immune-related biological processes. Additionally, DGCR5 showed a significant correlation with stromal and immune cell populations, inflammatory activities and immune checkpoints. Clinically, patients with low-expression level of DGCR5 exhibited a worse overall survival. Conclusion: DGCR5 expression is downregulated in glioma, and low DGCR5 independently predicts worse prognosis in glioma patients. Moreover, DGCR5 is significantly associated with immune response and immune infiltration. These findings suggest that DGCR5 is a promising immunotherapy target and a novel prognostic biomarker for glioma. Keywords: DGCR5, immune infiltration, immune checkpoint, prognostic biomarker, glioma

\section{Introduction}

Gliomas are the most common and aggressive primary brain tumors with poor prognosis despite maximal surgical resection and current chemo-radiotherapy. ${ }^{1,2}$ Based on the pathological features, gliomas can be classified as World Health Organization (WHO) grade I to IV. ${ }^{3}$ Clinically, patients with high-grade gliomas have significantly worse prognosis and increased fatality relative to those with lowgrade gliomas. Glioblastoma multiforme (GBM, WHO grade IV), which is the most lethal type of glioma, has a median survival of approximately only one year with generally poor responses to all therapies. The failure of conventional cancer therapy has encouraged researchers to explore new and more targeted therapy options.

It is acknowledged that tumor microenvironment (TME) plays a crucial role in supporting the malignant growth and progression of glioma. ${ }^{4,5}$ The TME is comprised of various components, including infiltrating immune cells, fibroblasts, endothelial cells and extracellular matrix. ${ }^{6-8}$ The interplay between TME and tumor cells may contribute to immune evasiveness and systemic invasiveness of malignant cells by producing cytokines, chemokines, and angiogenic molecules. Recently, immune checkpoint blockade therapy made great success in cancers. ${ }^{9,10}$ For example, 
immune checkpoint inhibitors targeting PD-1/PD-L1 have been clinically successful and revolutionized the treatment of a number of advanced cancers, including melanoma and non-small-cell lung cancer. ${ }^{11,12}$ Monoclonal antibodies directed against cytotoxic $\mathrm{T}$ lymphocyte-associated antigen-4 (CTLA-4), such as ipilimumab, yield considerable clinical benefit for patients with metastatic melanoma. ${ }^{13,14}$ This success has greatly promoted the researches on immunotherapy of glioma. ${ }^{15-17}$ However, current immunotherapies in glioma showed poor clinical efficacy. It is urgent to elucidate the interactions between tumor and immunity, and identify novel immunotherapeutic targets in glioma.

Long non-coding RNAs (lncRNAs), which are a novel class of non-protein-coding transcripts that are longer than 200 nucleotides and lack apparent open reading frames, play a critical role in the development and progression of cancers. ${ }^{18}$ Accumulating evidence indicates that lncRNAs can serve as potential biomarkers for cancers and have significant roles in immune response, immune cell differentiation and the regulation of related processes. In addition, IncRNAs can regulate inflammatory factors and inflammation-related genes to control inflammation. ${ }^{19}$ However, the involvement of lncRNAs in the complicated immune regulation still needs further exploration.

DiGeorge syndrome critical region gene 5 (DGCR5) is a novel lncRNA, which is significantly decreased in Huntington's disease neurodegeneration. ${ }^{20}$ Increasing evidence has revealed that DGCR5 is a suppressor of lung cancer, cervical cancer, gastric cancer and hepatocellular carcinoma progression. ${ }^{21-24}$ Moreover, down-regulation of DGCR5 correlates with poor prognosis in hepatocellular carcinoma and bladder cancer. ${ }^{25,26}$ A recent study reported that DGCR5 inhibited the proliferation, aggressiveness phenotypes, and EMT of glioblastoma cell. ${ }^{27}$ However, the role of DGCR5 in glioma has remained largely unknown. The present study is aimed to investigate the role and underlying mechanisms of DGCR5 in glioma progression.

In this study, we comprehensively investigated DGCR5 expression pattern, and its correlations with immune functions and prognosis of glioma patients based on data from TCGA and CGGA datasets. Moreover, we analyzed the associations of DGCR5 with infiltrated stromal and immune cells. This is the largest and comprehensive study characterizing DGCR5 expression in whole grade gliomas at the molecular and clinical levels through largescale analysis. Our results revealed that DGCR5 may be a promising biomarker and therapeutic target for glioma.

\section{Materials and Methods \\ Data Collection and Human Tissue Samples}

The TCGA dataset was obtained from the University of California, Santa Cruz, Xena browser (https://xenabrowser. net/), which contains 697 RNA-seq samples as well as clinical annotation. An additional dataset including 301 samples of mRNA microarray data was downloaded from Chinese Glioma Genome Atlas (CGGA) (http://www.cgga.org.cn/).

In addition, we obtained glioma tissue samples from 35 patients (10 grade II, 10 grade III, and 15 grade IV glioma) and 10 normal brain tissue samples from the Department of Neurosurgery, The Affiliated Wuxi No.2 People's Hospital of Nanjing Medical university, China. The normal brain tissue samples were obtained from 10 individuals undergoing internal decompression surgery following severe traumatic brain injury. This study was approved by the Ethics Committee of The Affiliated Wuxi No.2 People's Hospital of Nanjing Medical University and informed consent was obtained from all the patients.

\section{Cell Lines}

Human glioblastoma cells (LN229, A172, U251, and U87) were purchased from the Cell bank of the Chinese Academy of Sciences (Shanghai, China). The normal human astrocyte cell line (HA1800) was purchased from Boster Biological Technology, Ltd. (Wuhan, China). All the cell lines were cultured in DMEM containing $10 \%$ fetal bovine serum, $100 \mu \mathrm{g} / \mathrm{mL}$ penicillin and $100 \mu \mathrm{g} / \mathrm{mL}$ streptomycin and cultured at $37^{\circ} \mathrm{C}$ with $5 \% \mathrm{CO}_{2}$.

\section{Quantitative Real-Time PCR (qRT-PCR) Assay}

RNAs were extracted using Trizol reagent (Invitrogen) according to the manufacturer's protocol. Then, RNAs were transcribed into cDNAs using the PrimeScript RT Master Mix Perfect Real Time (TaKaRa, Otsu, Shiga, Japan). qRTPCR was carried out using SYBR Premix Ex Taq II (Takara Bio). $\beta$-actin mRNA was included as internal control.

\section{Functional Annotation of DGCR5}

Significantly related genes with DGCR5 expression were retrieved by using the Pearson correlation analysis. Gene ontology (GO) analysis was subsequently performed on DAVID Bioinformatics Resources 6.7 (http://david.abcc. ncifcrf.gov/). 


\section{Statistical Analysis}

$\mathrm{R}$ language was the main tool to generate figures and perform the statistical analysis. The overall survival difference was calculated with the Kaplan-Meier method by the survival package. Several R packages, such as ggplot2, pheatmap, pROC, circlize, corrplot and corrgram, were used to generate figures. SPSS 23.0 was used to examine prognostic factors by Cox proportional hazards regression model. For in vitro experiments, the statistical analysis was performed using GraphPad Prism version 7.0. A $p$-value less than 0.05 is considered statistically significant.

\section{Results}

\section{DGCR5 is Downregulated in Glioma}

To determine the differences of DGCR5 expression between glioma and normal brain tissues, we first analyzed the mRNA levels of DGCR5 using GEPIA. As shown in Figure 1A, DGCR5 expression was lower in LGG (lower grade glioma) and GBM compared with that in the normal tissues. We next examined the RNA-seq data from TCGA dataset to explore the expression pattern of DGCR5 in glioma. The results revealed that DGCR5 expression was markedly decreased in GBM compared with that in grade II and grade III gliomas (Figure 1B). This result was similar in the CGGA dataset (Figure 1C). To further validate these findings, we used qRT-PCR to detect the expression levels of DGCR5 in our glioma samples, normal brain tissues and cell lines. As shown in Figure 1D, DGCR5 expression was much lower in glioma tissues than that in normal brain tissues. In addition, the expression of DGCR5 was negatively correlated with WHO grade of gliomas (Figure 1E). Moreover, DGCR5 expression was downregulated in glioma cell lines (LN229, A172, U251, and U87) compared with that in normal astrocyte cell line (HA1800) (Figure 1F). These results demonstrated that DGCR5 expression is significantly downregulated in glioma.

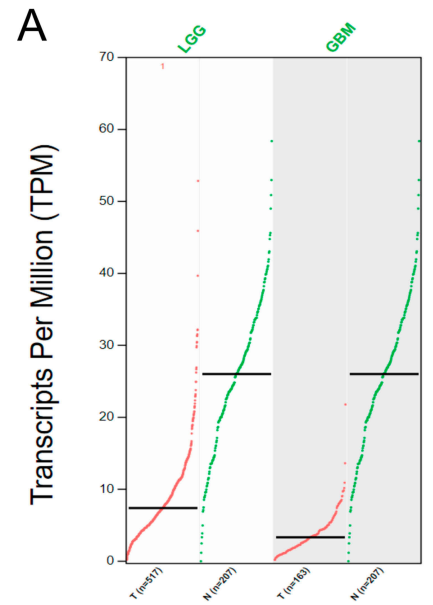

D

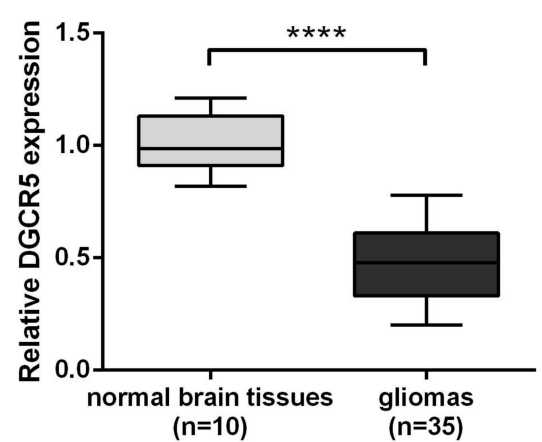

B

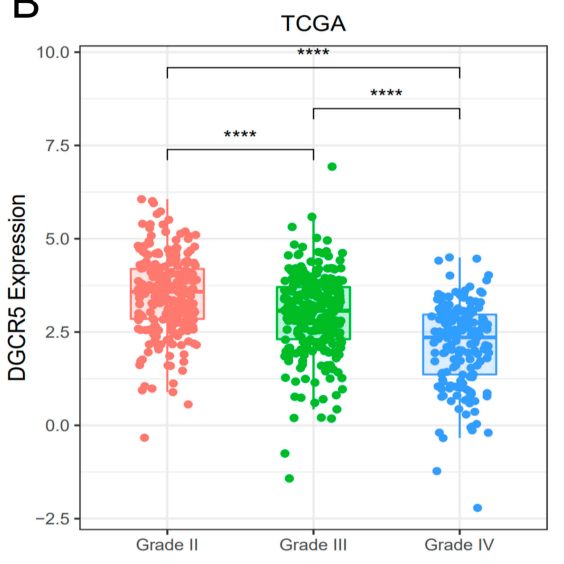

E

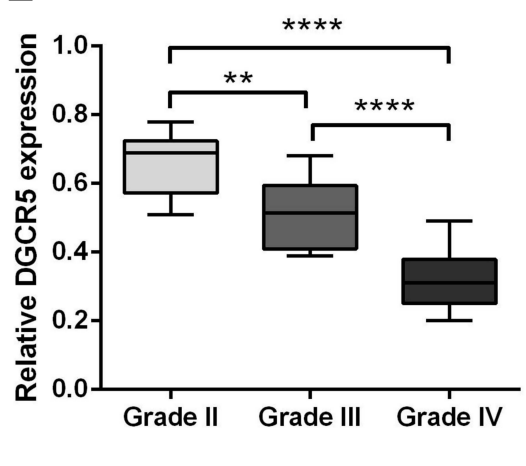

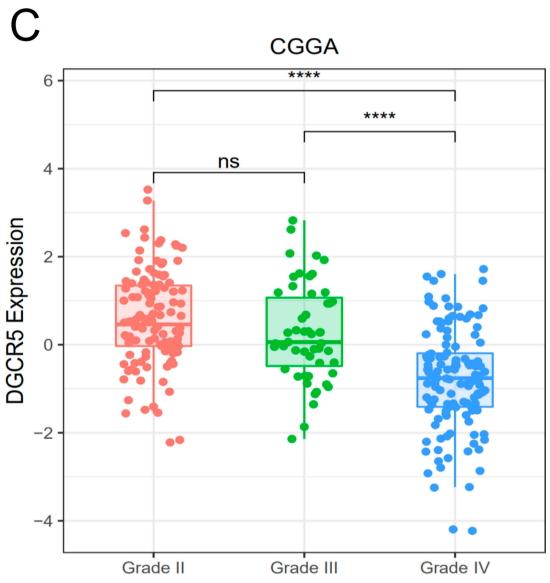

F

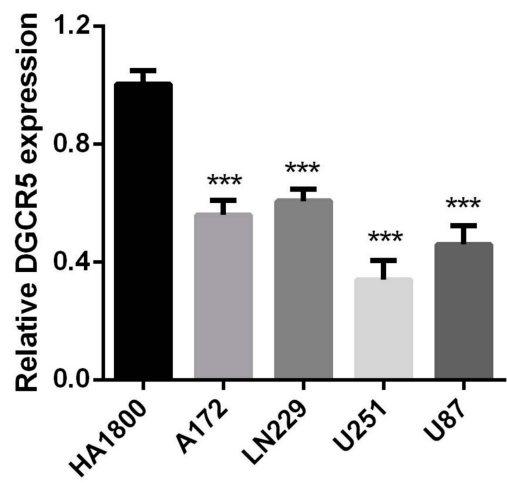

Figure I DGCR5 is significantly decreased in gliomas and negatively associated with tumor aggressiveness. (A) Differential expression of DGCR5 in brain lower grade glioma (LGG) and glioblastoma (GBM) compared to that in normal brain tissues in GEPIA. (B-C) DGCR5 expression in glioma of WHO grade II-IV based on TCGA and CGGA datasets. (D) Validation of aberrant mRNA expression of DGCR5 in 35 gliomas compared to that in 10 normal brain tissues via qRT-PCR. (E) DGCR5 expression in glioma of WHO grade II-IV based on our patient samples. (F) The mRNA expression level of DGCR5 in glioma cell lines and normal glial cell line by qRT-PCR. $* * \quad p<0.01$; $* * *$ $p<0.001 ; * * * * * 0.0001 ; n s$, not significant. 


\section{DGCR5 is Enriched in IDH Mutant}

\section{Glioma}

Emerging evidence has indicated that the IDH mutation status is remarkably related to clinical outcomes and oncogenic progression in glioma patients. ${ }^{28}$ Generally, IDH mutant glioma carries an improved prognosis compared with IDH wild-type glioma of similar grade. Thus, we further measured the correlation of DGCR5 expression with IDH status. We found that DGCR5 was highly enriched in IDH mutant glioma in both TCGA and CGGA datasets (Figure 2A-B). Subsequently, we performed ROC analysis to assess the diagnostic value of DGCR5 as a biomarker for detecting IDH status in glioma. The area under the curves (AUC) in TCGA and CGGA datasets were $71.4 \%$ and $69.7 \%$, respectively (Figure $2 \mathrm{C}-$ D). These data suggested that DGCR5 is significantly enriched in IDH mutant glioma.
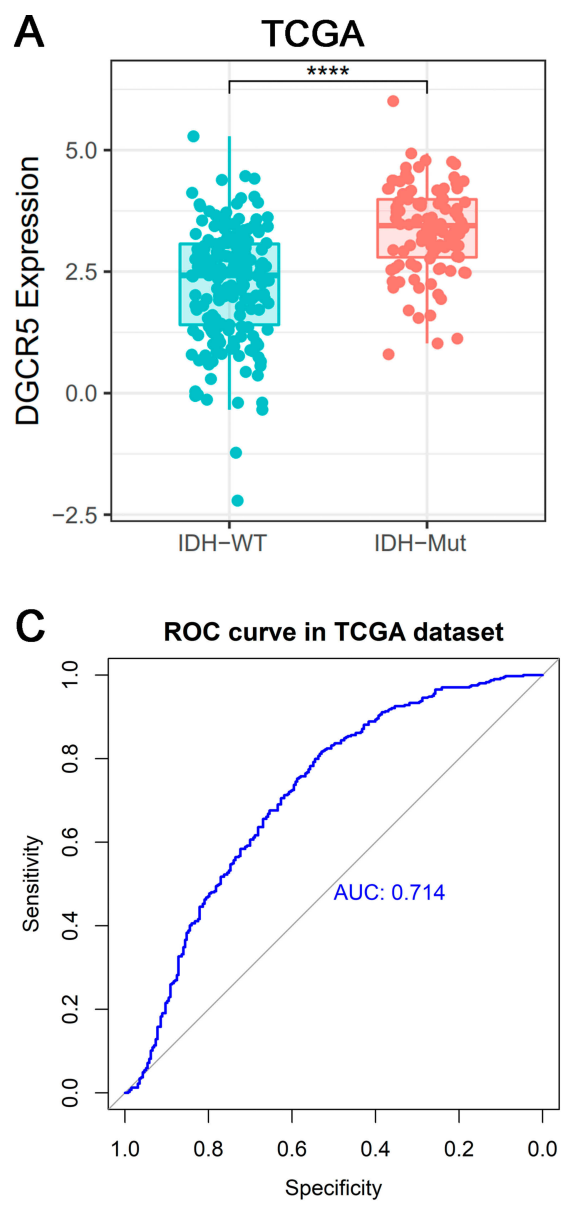

\section{DGCR5 is Related to Immune Functions} in Glioma

Since DGCR5 expression was distinctly associated with the malignancy of glioma, we inferred that DGCR5 had important biological functions in glioma progression. To identify the potential mechanisms of DGCR5, we first analyzed the related genes in the datasets by the Pearson correlation analysis $(|\mathrm{r}|$ $>0.4$ and $p<0.01$ ). Totally, 1770 and 2000 positive genes, and 1425 and 2056 negative genes were selected as significantly correlated with DGCR5 expression in TCGA and CGGA datasets, respectively. We then examined the biological functions of these genes on the DAVID website. GO analysis showed that genes negatively related to DGCR5 were significantly enriched in immune response, immune system process and other immune-related functions in both datasets (Figure $3 \mathrm{~A}-\mathrm{B}$ ). These findings suggested that DGCR5 may participate in the regulation of immune system in glioma.
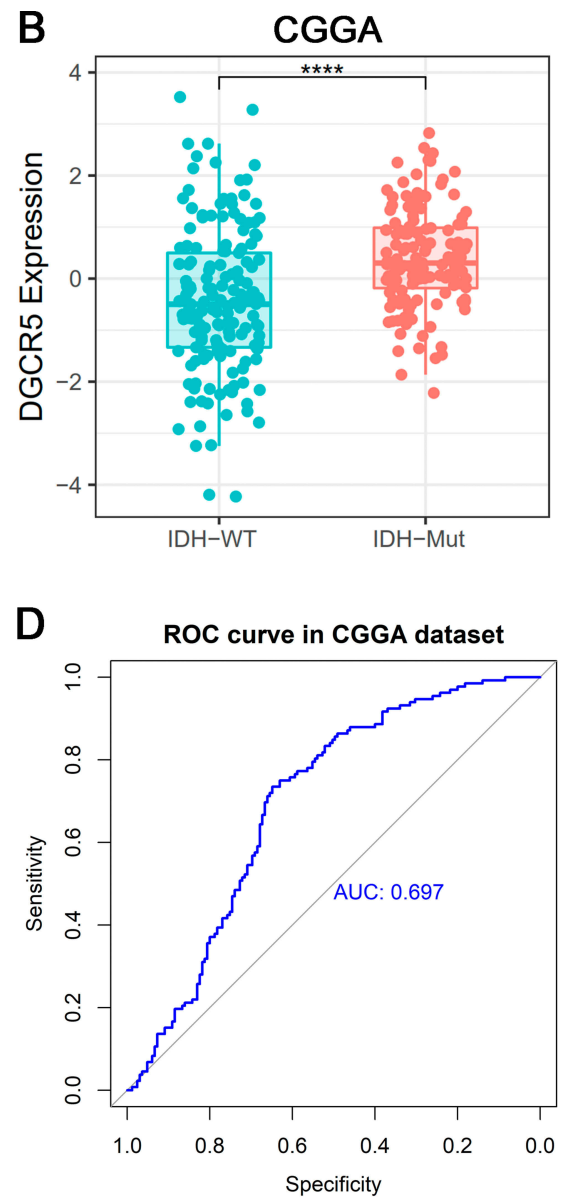

Figure 2 DGCR5 is enriched in IDH mutant glioma. (A, B) DGCR5 is significantly overexpressed in IDH mutant glioma in TCGA and CGGA datasets. (C, D) ROC curve analysis reveals the predictive value of DGCR5 for IDH mutant glioma in TCGA and CGGA datasets. **** $p<0.000$ I. 
A

Top 8 of GO Enrichment in TCGA

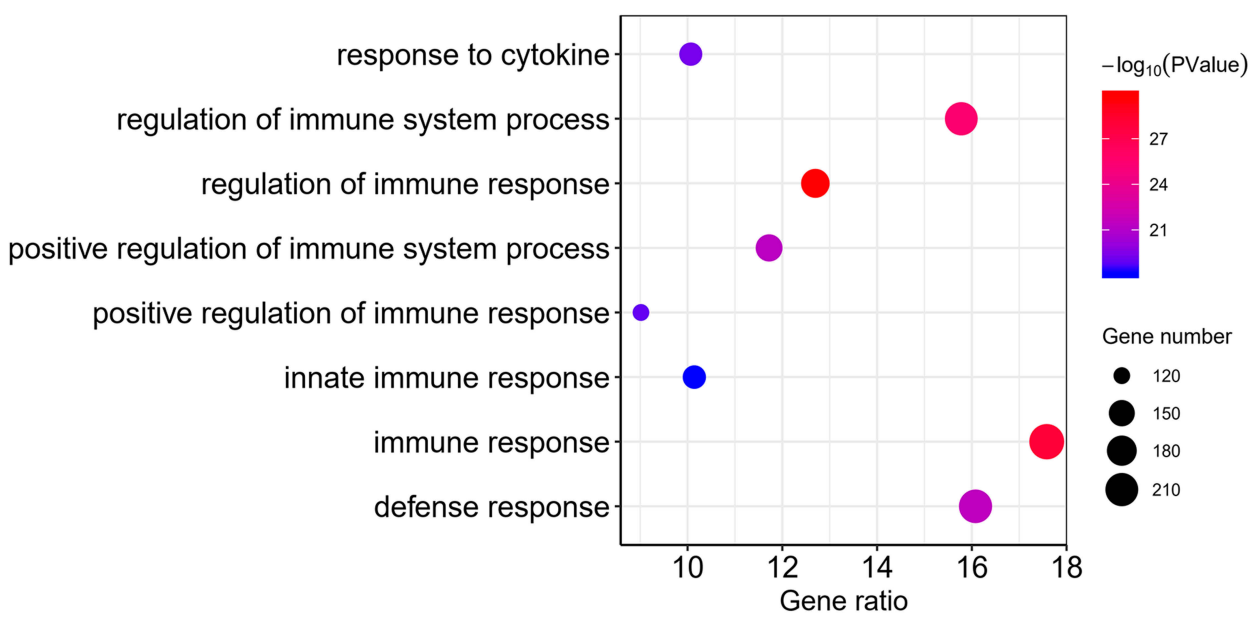

B

\section{Top 8 of GO Enrichment in CGGA}

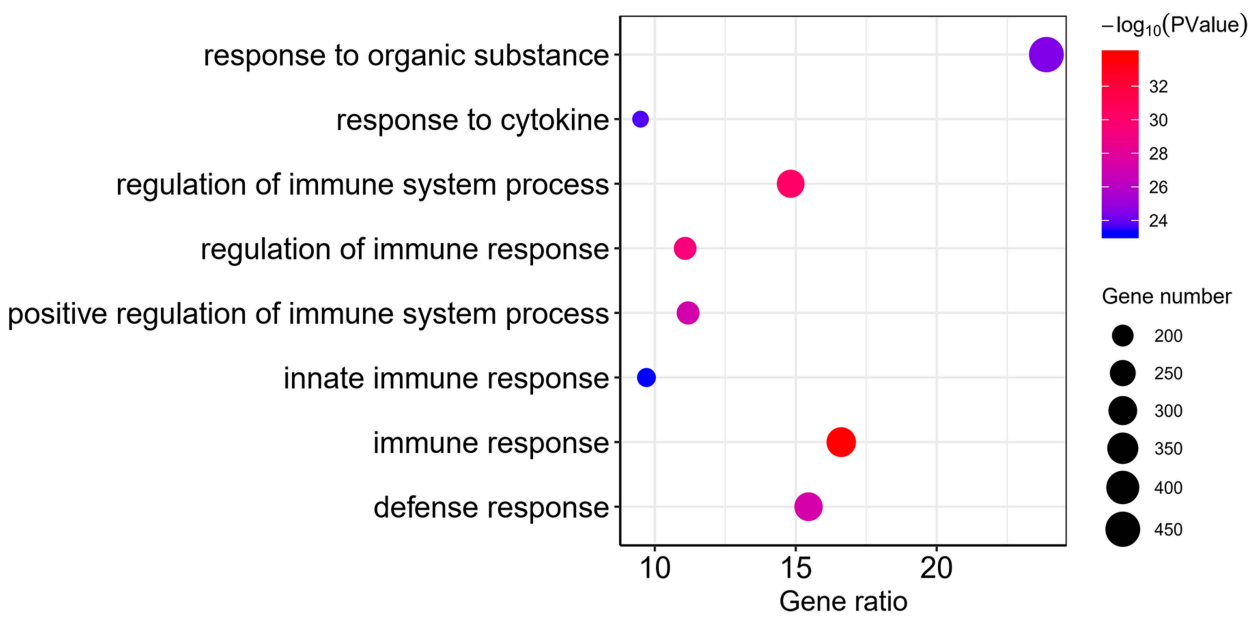

Figure 3 DGCR5 is related to immune functions in glioma. (A, B) GO analysis shows that DGCR5 is involved in immune response, immune system process and other immune-related functions in both TCGA and CGGA datasets.

\section{DGCR5 is Related to Inflammatory Activities in Glioma}

To explore whether DGCR5 is associated with inflammatory response, we selected seven clusters (comprising 104 genes in total), which were previously established as metagenes representing different types of immune response functions. ${ }^{29}$ Most genes were negatively correlated with DGCR5 expression except for IgG (Figure 4A-B). To verify the above findings, Gene Sets Variation Analysis (GSVA) was used to transform gene expression into enrichment scores for these metagenes. Corrgrams were obtained based on the Pearson's $r$ values between DGCR5 and seven metagenes (Figure 4C-D). We found that
DGCR5 was negatively associated with HCK, LCK, MHC-I, MHC-II, STAT1 and interferon, in consistent with what we observed in Figure 4A-B. These results indicated that DGCR5 may play a crucial role in inflammatory response.

\section{DGCR5 is Correlated with Infiltrated} Cells in Glioma Microenvironment

To get a better understanding of the relationship between DGCR5 and the infiltrated cells, we used ESTIMATE algorithm method described by Yoshihara. ${ }^{30}$ As shown in Figure 5A-B, in both TCGA and CGGA datasets, DGCR5 expression was significantly negatively associated with stromal 


\section{A DGCR5-related inflammatory activities in TCGA}

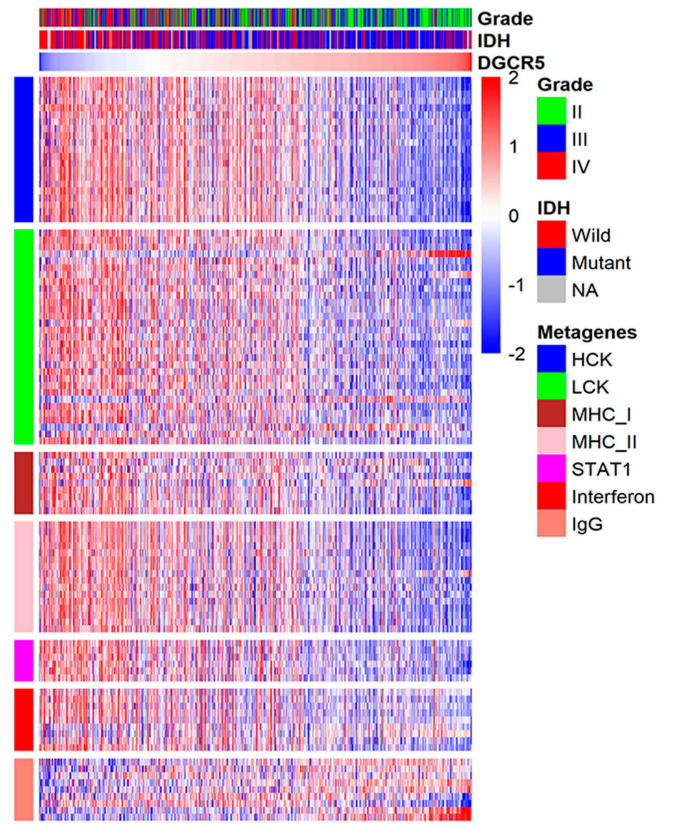

C

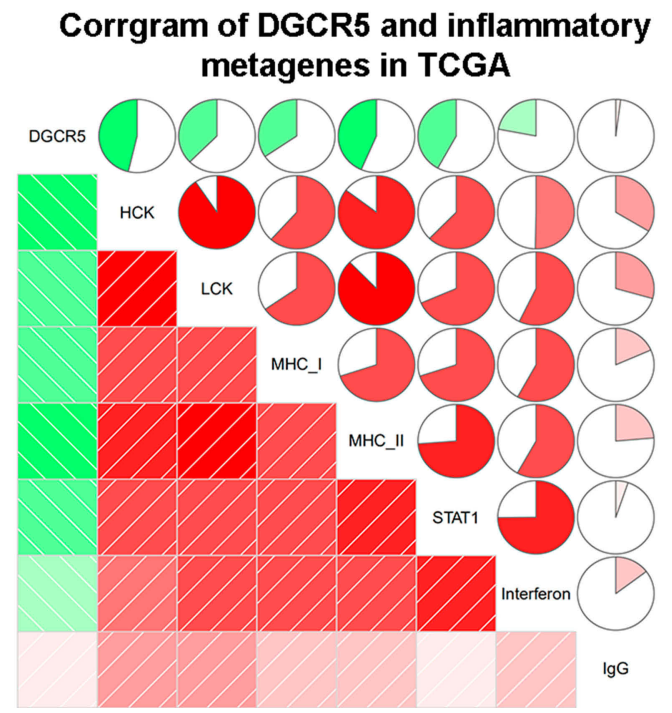

B DGCR5-related inflammatory activities in CGGA

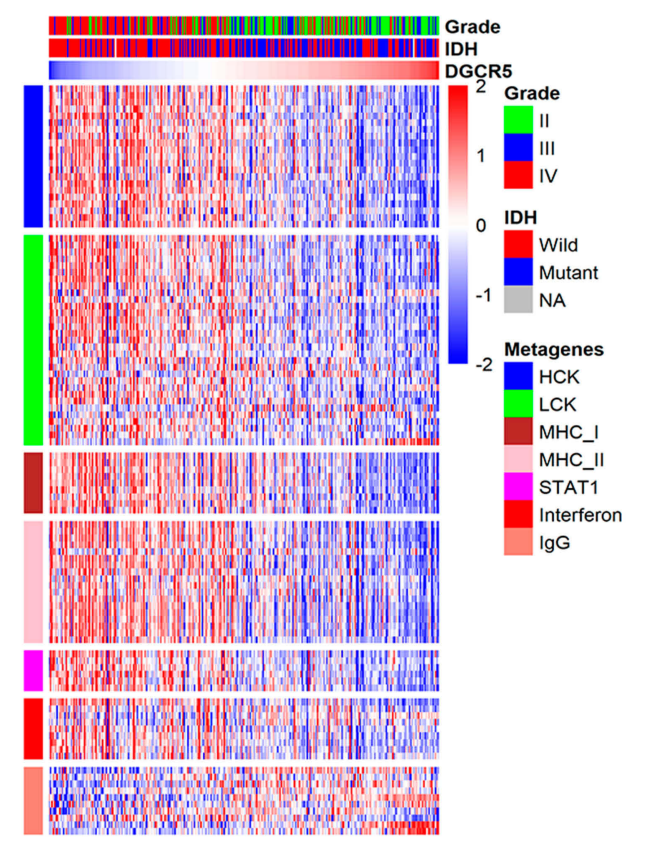

D

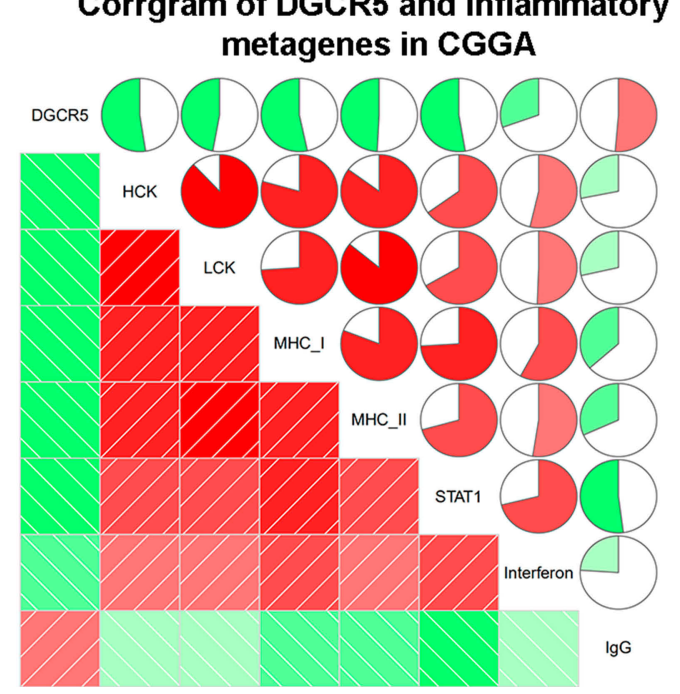

Figure 4 The relationship between DGCR5 and immune activities. (A, B) Heatmaps displaying the clinicopathological parameters, DGCR5 expression, and seven related metagenes from TCGA and CGGA datasets. (C, D) Corrgrams were established based on the relationship between DGCR5 expression and GSVA enrichment scores for these seven metagenes.

score, immune score, and ESTIMATE score, suggesting that DGCR5 has a remarkable influence on the infiltration level of stromal and immune cell. Furthermore, Microenvironment Cell Populations-counter (MCP) method was used to evaluate the relationship between DGCR5 and specific cell populations in the TME of glioma. ${ }^{31} \mathrm{We}$ found that DGCR5 showed a strong negative correlation with monocytic lineage, fibroblasts, myeloid dendritic cells, neutrophils and T cells (Figure 5A-B). To further determine these findings, we next employed $\mathrm{xCell}^{32}$ to analyze the correlation between DGCR5 expression and 64 immune and stromal cell types. The results revealed that DGCR5 expression was remarkably negatively correlated with 34 cell types, whereas positively correlated with 14 cell types (Figure 6). These cell types comprised 12 lymphoid, 12 myeloid cells, 11 stromal, six stem, and seven other cell types. Notably, DGCR5 expression was negatively correlated with the majority of myeloid cells and stromal cells. While the expression of DGCR5 was 


\section{A}

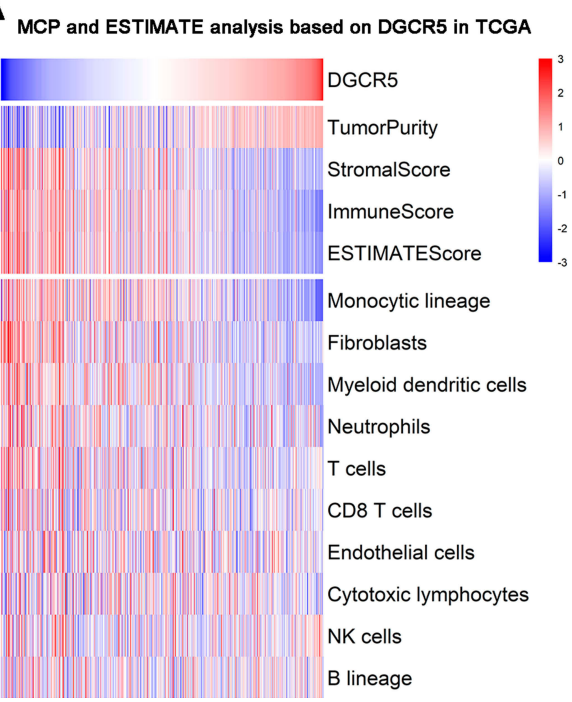

\section{B}

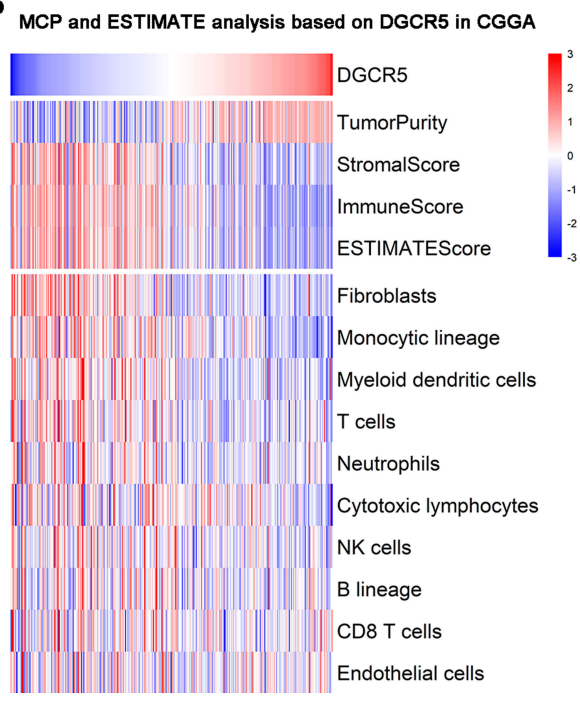

Figure 5 DGCR5 is tightly associated with immune score and infiltrated cells in the glioma microenvironment. (A-B, upper panel) DGCR5 is negatively associated with immune score and stromal score. (A-B, lower panel) DGCR5 is closely associated with infiltrated cells in the tumor microenvironment.

positively associated with mesenchymal stem cells (MSCs), suggested that DGCR5 has an important influence on the CD8+ T cells and eosinophils, which were commonly recognized as anti-tumor cells. ${ }^{33-35}$ These results strongly glioma. infiltration of stromal and immune cells in the TME of

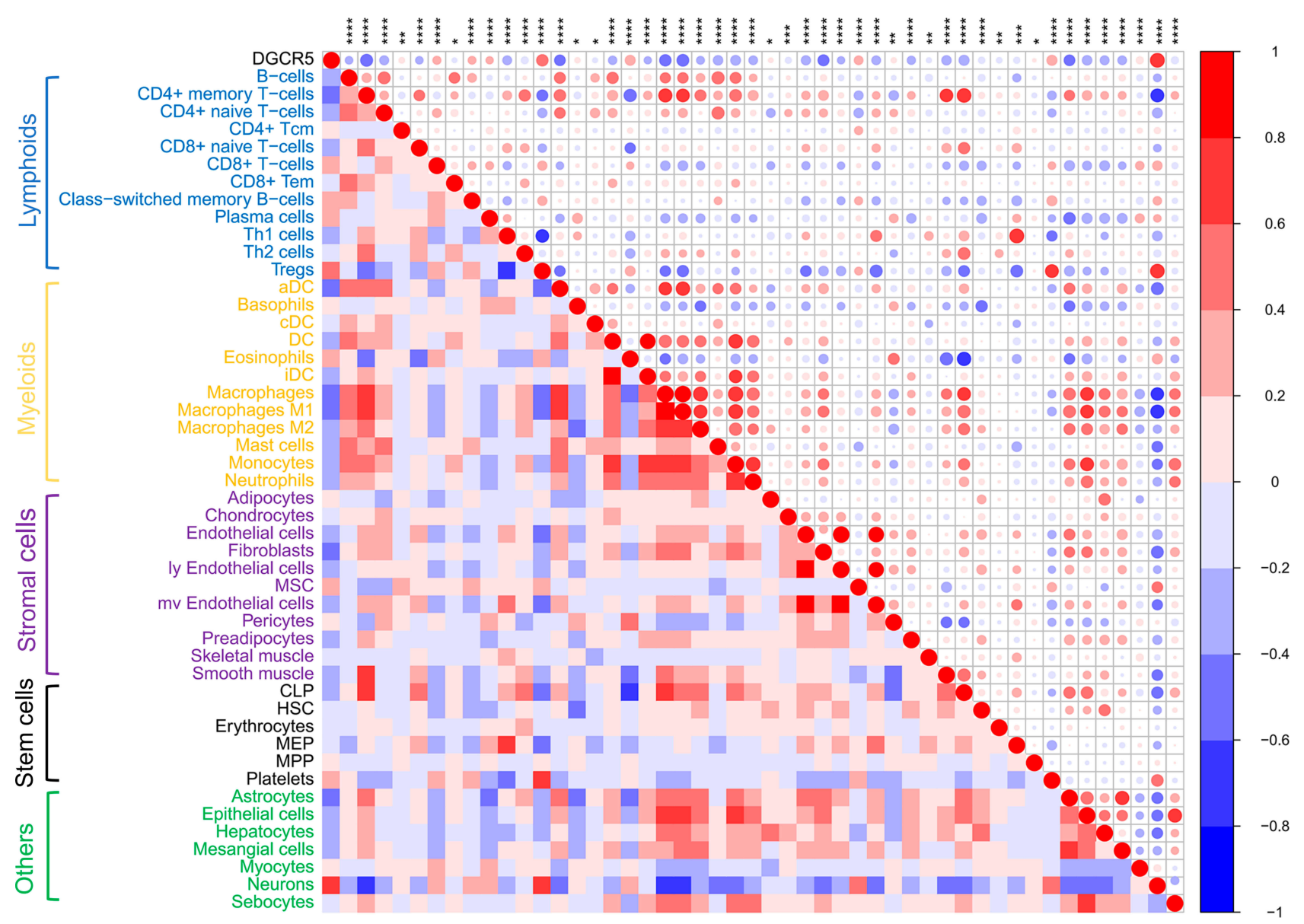

Figure 6 Correlation between DGCR5 expression and xCell scores in glioma. ${ }^{*} p<0.05 ; * * p<0.01$; $* * * p<0.001 ; * * * * p<0.000$ I. 


\section{DGCR5 is Negatively Associated with} Immune Checkpoints and

\section{Immunosuppressive Properties}

Emerging studies have demonstrated that immune checkpoint members can act as therapeutic targets in clinical application or clinical trial. ${ }^{36}$ Therefore, we analyzed the relationship between DGCR5 and the currently available immune checkpoint blocking molecules, including PD-1

A

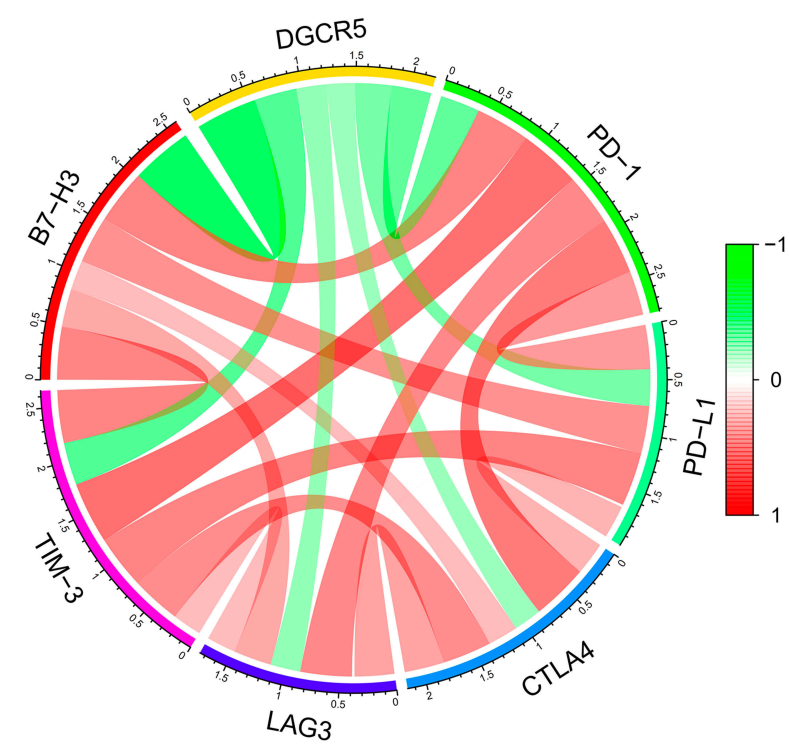

C corrplot of DGCR5 and immunosuppressive cell recuritment factors

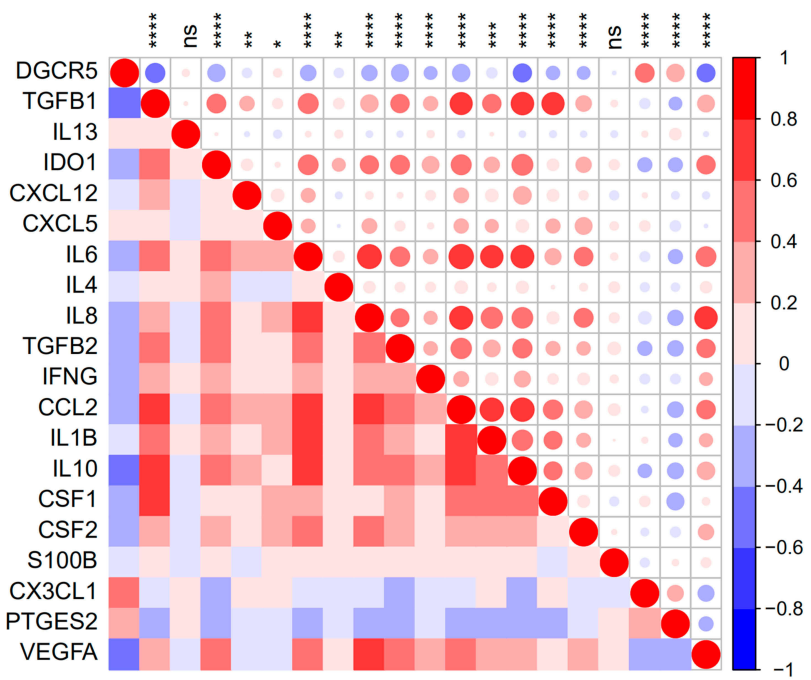

(PDCD1)，PD-L1 (CD274), CTLA-4, LAG3, TIM-3 (HAVCR2) and B7-H3 (CD276). Pearson correlation analysis showed that DGCR5 was notably negatively associated with these immune checkpoints in glioma (Figure 7A-B), indicating the potential regulatory effects of DGCR5 on these checkpoint members.

Since DGCR5 is significantly related to immunosuppressive cells, such as macrophages, neutrophils and dendritic cells, we speculated that DGCR5 may be related to
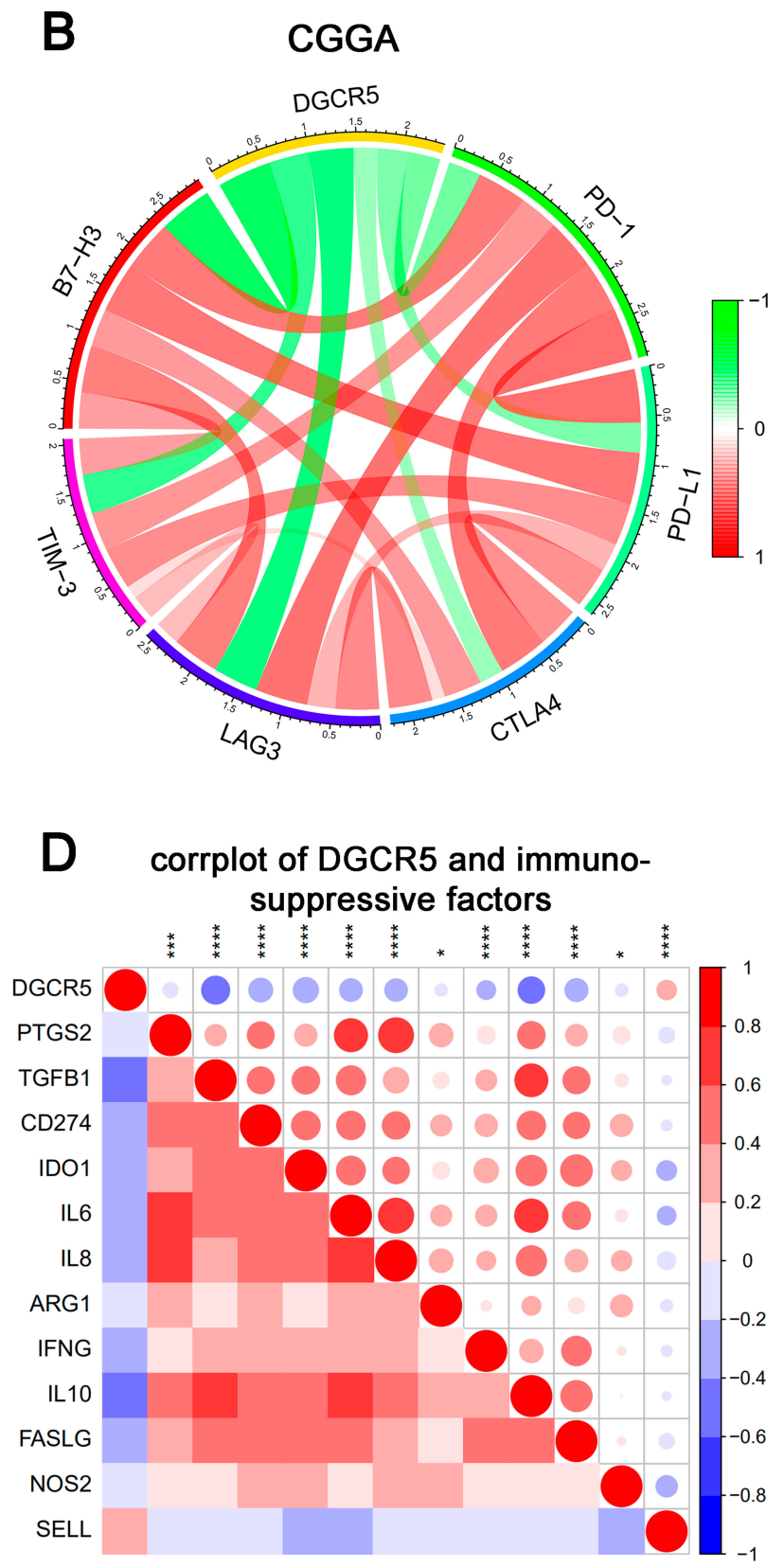

Figure 7 DGCR5 correlates with immune checkpoints and immunosuppressive properties. (A, B) Correlation between DGCR5 and immune checkpoints (PD-I, PD-LI, CTLA4, LAG3, TIM-3 and B7-H3) in TCGA and CGGA datasets. (C) Correlation between DGCR5 and immunosuppressive cell recruitment factors. (D) Correlation between DGCR5 and immunosuppressive factors secreted by myeloid-derived suppressor cells (MDSCs), tumor-associated macrophages (TAMs) and tumor-associated neutrophils (TANs). ${ }^{*} p<0.05$; $* * 0<0.01$; *** $p<0.00$ I; **** $p<0.000$ I; ns, not significant. 
the immunosuppressive properties of glioma. To confirm this, we selected critical factors that recruit myeloidderived suppressor cells, tumor-associated macrophages (TAMs), and tumor-associated neutrophils, as well as the immunosuppressive factors secreted by these cells, and then performed correlation analysis to analyze the relationship between DGCR5 expression and these factors. ${ }^{37}$ Surprisingly, DGCR5 showed an obviously negative correlation with the majority of immunosuppressive cell recruitment factors and immunosuppressive factors (Figure 7C-D). These findings were consistent with the above results and further confirmed the potential immunotherapeutic value of DGCR5 in glioma.

\section{DGCR5 is an Independent Prognostic Biomarker for Glioma Patients}

We next investigated the prognostic value of DGCR5 in glioma. As shown in Figure 8A-B, glioma patients with low DGCR5 expression had a significantly shorter survival than patients with high DGCR5 expression in both TCGA and CGGA datasets. To further determine this result, we conducted univariate and multivariate Cox regression analyses in TCGA dataset (Table 1). In the univariate analysis, DGCR5 expression as well as some other factors including age, KPS, tumor grade, radiation and targeted molecular therapy were significantly associated with overall survival of glioma patients. Next, the multivariate Cox regression analysis incorporating DGCR5 expression, age, KPS, tumor grade, radiation and targeted molecular therapy revealed that DGCR5 expression remained an independent prognostic factor for glioma patients after taking other factors into consideration.

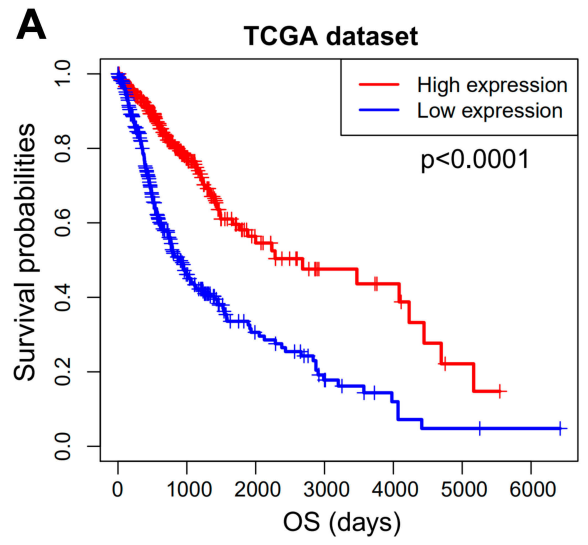

\section{Discussion}

Glioma is the most prevalent and lethal type of central nervous system (CNS) tumor in adults, representing approximately $50 \%$ of all brain tumors. Improvements in treatment are very limited despite the developments in conventional therapies, including neurosurgical resection, adjuvant chemotherapy, radiotherapy and targeted therapy. Considering the extremely poor outcome and high resistance to standard treatment, new therapeutic approaches are urgently needed. Recent advances in immunotherapy have shown a promising prospect for glioma. ${ }^{38,39}$ Particularly, immune checkpoint blockade has made great progress in treatment of glioma. For instance, combination therapy with 4-1BB activation and CTLA-4 blockade in the setting of focal radiation therapy improves survival in an orthotopic mouse model of glioma. ${ }^{40}$ Anti-PD-1/PD-L1 combined with radiotherapy also improved local control and overall survival of glioma. ${ }^{41}$ However, many factors limit the efficiency of current immunotherapy in treating glioma, because glioma has a low immunogenic response and an immunosuppressive microenvironment caused by the precise crosstalk between cytokines and immune cells. ${ }^{42}$ Hence, it is worth to explore new immunotherapeutic targets to cure glioma.

Accumulating studies have shown the importance of lncRNAs in the regulation of immune cell differentiation, immune and inflammatory responses. ${ }^{43}$ LncRNA DGCR5 is first reported to be decreased in Huntington's disease. ${ }^{20}$ Moreover, DGCR5 has been reported to function as a tumor suppressor in papillary thyroid carcinoma, ${ }^{44}$ hepatocellular carcinoma ${ }^{45}$ and gastric cancer ${ }^{23}$ via acting as a competing endogenous RNA. However, DGCR5 may act as an oncogene in some tumors. For instance, DGCR5 is upregulated in lung adenocarcinoma (LUAD), and inhibition

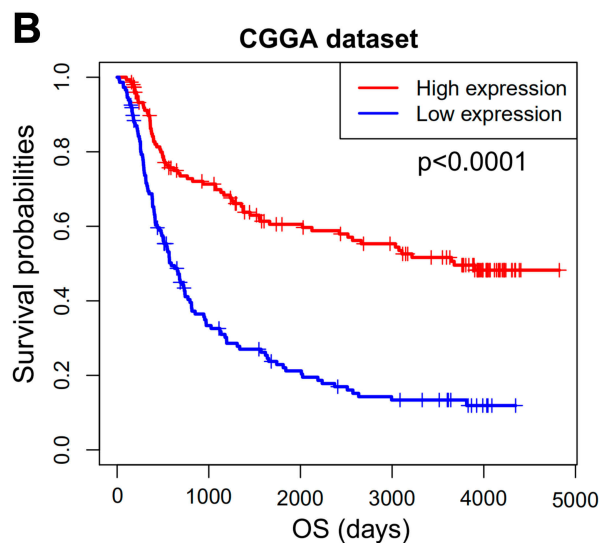

Figure 8 Survival analysis of DGCR5 in glioma. (A, B) Low expression of DGCR5 is associated with worse overall survival of glioma patients based on TCGA and CGGA datasets. 
Table I Univariate and Multivariate Cox Regression Analyses of Various Clinicopathological Characteristics in Relation to Overall Survival in Glioma Patients from TCGA Cohort

\begin{tabular}{|c|c|c|c|c|c|c|}
\hline \multirow[t]{2}{*}{ Characteristic } & \multicolumn{3}{|c|}{ Univariate Analysis } & \multicolumn{3}{|c|}{ Multivariate Analysis } \\
\hline & HR & $95 \% \mathrm{Cl}$ & p-value & HR & $95 \% \mathrm{Cl}$ & p-value \\
\hline Age ( $\geq 47 y$ vs $<47 y$ ) & 4.299 & $3.247-5.693$ & $<0.001$ & 1.914 & $1.297-2.824$ & 0.001 \\
\hline Gender (Male vs Female) & 1.186 & $0.922-1.527$ & 0.184 & & & \\
\hline KPS score (>80 vs $\leq 80)$ & 0.297 & $0.220-0.402$ & $<0.001$ & 0.641 & $0.437-0.940$ & 0.023 \\
\hline Tumor grade (GBM vs LGG) & 8.932 & $6.757-11.807$ & $<0.001$ & 5.371 & $3.436-8.397$ & $<0.001$ \\
\hline Radiation (yes vs no) & 2.301 & $1.636-3.235$ & $<0.001$ & 0.909 & $0.567-1.456$ & 0.690 \\
\hline Targeted molecular therapy (yes vs no) & 0.632 & $0.486-0.823$ & 0.001 & 1.432 & $1.010-2.031$ & 0.044 \\
\hline DGCR5 expression (Low vs High) & 2.556 & $1.955-3.342$ & $<0.001$ & 1.598 & I.123-2.273 & 0.009 \\
\hline
\end{tabular}

Abbreviations: KPS, Karnofsky Performance Scale; LGG, lower grade glioma; GBM, glioblastoma multiforme; TCGA, The Cancer Genome Atlas.

of DGCR5 can prevent LUAD progression via playing antiapoptosis roles. ${ }^{46}$ Knockdown of DGCR5 suppresses laryngeal carcinoma progression and enhances the radiosensitivity of human laryngeal carcinoma cells via inducing miR-195. ${ }^{47}$ In this study, we found that DGCR5 expression was abnormally lower in glioma, and also significantly associated with glioma grade. It is well known that IDH mutation has an important influence on the development and progression of glioma. This study also showed that DGCR5 is highly enriched in IDH mutant glioma. In addition, we reported that increased levels of DGCR5 expression are associated with better prognosis in glioma, which is consistent with that IDH mutant glioma generally has a longer overall survival than IDH wild-type glioma.

Immunotherapy is the most promising treatment for malignant tumors. However, the TME, including stromal cells, inflammatory cells, vasculature, and extracellular matrix, usually participates in supporting the progression of glioma. Thus, it is urgently needed to discover new molecular biomarkers and targets that play vital roles in the TME. Here, we found that DGCR5 plays a crucial role in immune response, immune system process and defense response, as well as significantly correlates with infiltrated stromal and immune cells. In addition, DGCR5 is significantly negatively correlated with immunosuppressive properties of glioma, such as cytokines or chemokines that recruit immunosuppressive cells, including myeloidderived suppressor cells, TAMs, and tumor-associated neutrophils, as well as immunosuppressive factors secreted by these cells. The correlations between DGCR5 expression and marker genes encoding immunoregulatory factors imply a role for DGCR5 in regulating tumor immunology in glioma. These special characteristics of DGCR5 have not been reported in previous studies.
Immune checkpoint inhibitors, which are the most promising approaches to activate antitumor immune response, have made great success in treatment of various cancers. ${ }^{48,49}$ However, some cancer patients may have immune-related adverse events from the treatment of immune checkpoint blockades. ${ }^{50}$ In recent years, accumulating studies have demonstrated a combination of immune checkpoint blockades, which may be a promising therapy for glioma. ${ }^{51,52}$ Considering the significant efficacy of immune checkpoint blockade therapy, we further evaluated the correlations between DGCR5 and immune checkpoints in glioma. Our results showed that DGCR5 is negatively associated with the checkpoint proteins PD-1, PD-L1, CTLA-4, LAG3, TIM-3 and B7-H3, indicating the potential effect of DGCR5 on regulating these immune checkpoints.

Mechanistically, DGCR5 might regulate the immunity in glioma through $\mathrm{Wnt} / \beta$-catenin signaling pathway. Previous research works have reported that overexpression of DGCR5 inhibited Wnt/ $\beta$-catenin signaling in cancers, ${ }^{22,53,54}$ indicating that the role of DGCR5 on regulating tumor progression may be based on this classical pathway. In addition, increasing evidence suggests that dysregulated $\mathrm{Wnt} / \beta$-catenin signaling may support malignant transformation and disease progression through a variety of mechanisms in the TME. ${ }^{55}$ $\mathrm{Wnt} / \beta$-catenin signaling positively or negatively affects anticancer immunosurveillance by modulating various aspects of tumor-immune cell interactions including the immunogenicity of cancer cells and the ability of immune cells, such as dendritic cells (DCs), natural killer (NK) cells, Treg cells, myeloid-derived suppressor cells (MDSCs), and cytotoxic T lymphocytes (CTLs) to elicit effective tumortargeting immune responses. ${ }^{55}$ For example, activation of tumor cell-intrinsic $\beta$-catenin prevents spontaneous $\mathrm{T}$ cell priming and infiltration into the TME. ${ }^{56,57}$ Moreover, tumor- 
intrinsic $\beta$-catenin activity contributes to exclusion of T-cell infiltration into the colorectal cancer TME by regulating CCL4 expression to recruit CD103 DCs. ${ }^{58}$ Furthermore, Wnt/ $\beta$-catenin signaling participates in the modulation of PD-L1 in triple-negative breast cancer, perhaps to help cancer cells escape immune surveillance. ${ }^{59}$ In the absence of Wnt ligands, GSK3 $\beta$ mediates the phosphorylationdependent proteasomal degradation of PD-L1 by $\beta$-TrCP, and tumor infiltration is ultimately increased by interferonproducing CTLs. ${ }^{60}$ Additionally, Wnt/ $\beta$-catenin signaling induces the expression of CTLA-4 in human melanoma tumors through the activity of $\mathrm{TCF} / \mathrm{LEF}$ transcription factors. ${ }^{61}$ Based on these theories, we hypothesized that the interaction between DGCR5 and TME may be contributed by regulating the $\mathrm{Wnt} / \beta$-catenin signaling pathway. However, further molecular biological experiments are required to validate this hypothesis.

\section{Conclusion}

In conclusion, we explored the expression, clinical characteristics and biological functions of DGCR5 in glioma. Although the exact mechanisms of DGCR5 still require further investigation, our results indicate that DGCR5 may act as a potential therapeutic target in immunotherapy of glioma.

\section{Funding}

This work was supported by the Medical innovation Team Projects of Jiangsu Province ([2017]4).

\section{Disclosure}

The authors report no conflicts of interest in this work.

\section{References}

1. Buckner JC, Brown PD, O’Neill BP, Meyer FB, Wetmore CJ, Uhm JH. Central nervous system tumors. Mayo Clin Proc. 2007;82 (10):1271-1286. doi:10.4065/82.10.1271

2. Siegel R, Ma J, Zou Z, Jemal A. Cancer statistics, 2014. CA Cancer J Clin. 2014;64(1):9-29. doi:10.3322/caac.21208

3. Louis DN, Perry A, Reifenberger G, et al. The 2016 World Health Organization classification of tumors of the central nervous system: a summary. Acta Neuropathol. 2016;131(6):803-820. doi:10.1007/ s00401-016-1545-1

4. Quail DF, Bowman RL, Akkari L, et al. The tumor microenvironment underlies acquired resistance to CSF-1R inhibition in gliomas. Science. 2016;352(6288):aad3018. doi:10.1126/science.aad3018

5. Quail DF, Joyce JA. Microenvironmental regulation of tumor progression and metastasis. Nat Med. 2013;19(11):1423-1437. doi:10.1038/ nm.3394

6. Shi Y, Ping Y-F, Zhou W, et al. Tumour-associated macrophages secrete pleiotrophin to promote PTPRZ1 signalling in glioblastoma stem cells for tumour growth. Nat Commun. 2017;8(1):15080. doi:10.1038/ncomms 15080
7. Nusblat LM, Carroll MJ, Roth CM. Crosstalk between M2 macrophages and glioma stem cells. Cell Oncol (Dordr). 2017;40 (5):471-482. doi:10.1007/s13402-017-0337-5

8. Rape A, Ananthanarayanan B, Kumar S. Engineering strategies to mimic the glioblastoma microenvironment. Adv Drug Deliv Rev. 2014;79-80:172-183. doi:10.1016/j.addr.2014.08.012

9. Ribas A, Wolchok JD. Cancer immunotherapy using checkpoint blockade. Science (New York, NY). 2018;359(6382):1350-1355.

10. Patel SA, Minn AJ. Combination cancer therapy with immune checkpoint blockade: mechanisms and strategies. Immunity. 2018;48 (3):417-433. doi:10.1016/j.immuni.2018.03.007

11. Mahoney KM, Freeman GJ, McDermott DF. The next immune-checkpoint inhibitors: PD-1/PD-L1 blockade in melanoma. Clin Ther. 2015;37(4):764-782. doi:10.1016/j.clinthera.2015.02.018

12. He J, Hu Y, Hu M, Li B. Development of PD-1/PD-L1 pathway in tumor immune microenvironment and treatment for non-small cell lung cancer. Sci Rep. 2015;5(1):13110. doi:10.1038/srep13110

13. Van Allen EM, Miao D, Schilling B, et al. Genomic correlates of response to CTLA-4 blockade in metastatic melanoma. Science (New York, NY). 2015;350(6257):207-211. doi:10.1126/science. aad0095

14. Hodi FS, O’Day SJ, McDermott DF, et al. Improved survival with ipilimumab in patients with metastatic melanoma. $N$ Engl J Med. 2010;363(8):711-723. doi:10.1056/NEJMoa1003466

15. Jouanneau E, Black KL, Veiga L, et al. Intrinsically de-sialylated CD103(+) CD8 T cells mediate beneficial anti-glioma immune responses. Cancer Immunol Immunother. 2014;63(9):911-924. doi:10.1007/s00262-014-1559-2

16. Qian J, Luo F, Yang J, et al. TLR2 promotes glioma immune evasion by downregulating MHC Class II molecules in microglia. Cancer Immunol Res. 2018;6(10):1220-1233. doi:10.1158/2326-6066.CIR18-0020

17. Qian J, Wang C, Wang B, et al. The IFN-gamma/PD-L1 axis between $\mathrm{T}$ cells and tumor microenvironment: hints for glioma anti-PD-1/PDL1 therapy. J Neuroinflammation. 2018;15(1):290. doi:10.1186/ s12974-018-1330-2

18. Okugawa Y, Toiyama Y, Hur K, et al. Metastasis-associated long non-coding RNA drives gastric cancer development and promotes peritoneal metastasis. Carcinogenesis. 2014;35(12):2731-2739. doi:10.1093/carcin/bgu200

19. Collier SP, Collins PL, Williams CL, Boothby MR, Aune TM. Cutting edge: influence of Tmevpg1, a long intergenic noncoding RNA, on the expression of Ifng by Th1 cells. J Immunol. 2012;189 (5):2084-2088. doi:10.4049/jimmunol.1200774

20. Johnson R. Long non-coding RNAs in Huntington's disease neurodegeneration. Neurobiol Dis. 2012;46(2):245-254. doi:10.1016/j. nbd.2011.12.006

21. Wang R, Dong H-X, Zeng J, Pan J, Jin X-Y. LncRNA DGCR5 contributes to CSC-like properties via modulating miR-330-5p/ CD44 in NSCLC. J Cell Physiol. 2018;233(9):7447-7456. doi:10.1002/jcp. 26590

22. Liu Y, Chang Y, Lu S, Xiang -Y-Y. Downregulation of long noncoding RNA DGCR5 contributes to the proliferation, migration, and invasion of cervical cancer by activating Wnt signaling pathway. J Cell Physiol. 2019;234(7):11662-11669. doi:10.1002/jcp.27825

23. Xu Y, Zhang G, Zou C, et al. Long noncoding RNA DGCR5 suppresses gastric cancer progression by acting as a competing endogenous RNA of PTEN and BTG1. J Cell Physiol. 2019;234 (7):11999-12010. doi:10.1002/jcp.27861

24. Wang X-L, Shi M, Xiang T, Bu Y-Z. Long noncoding RNA DGCR5 represses hepatocellular carcinoma progression by inactivating Wnt signaling pathway. J Cell Biochem. 2019;120(1):275-282.

25. Huang R, Wang X, Zhang W, et al. Down-regulation of LncRNA DGCR5 correlates with poor prognosis in hepatocellular carcinoma. Cell Physiol Biochem. 2016;40(3-4):707-715. doi:10.1159/0004 52582 
26. Fang C, He W, Xu T, Dai J, Xu L, Sun F. Upregulation of IncRNA DGCR5 correlates with better prognosis and inhibits bladder cancer progression via transcriptionally facilitating P21 expression. J Cell Physiol. 2019;234(5):6254-6262. doi:10.1002/jcp.27356

27. Y F, Huang Y-L. DGCR5 suppresses the EMT of pediatric primary glioblastoma multiforme cell and serves as a prognostic biomarker. Eur Rev Med Pharmacol Sci. 2019;23(22):10024-10034. doi:10.26355/eurrev_201911_19569

28. Huse JT, Aldape KD. The evolving role of molecular markers in the diagnosis and management of diffuse glioma. Clin Cancer Res. 2014;20(22):5601-5611. doi:10.1158/1078-0432.CCR-14-0831

29. Rody A, Holtrich U, Pusztai L, et al. T-cell metagene predicts a favorable prognosis in estrogen receptor-negative and HER2-positive breast cancers. Breast Cancer Res. 2009;11(2):R15. doi:10.1186/bcr2234

30. Y K, M S, E M, et al. Inferring tumour purity and stromal and immune cell admixture from expression data. Nat Commun. 2013;4:2612. doi: $10.1038 /$ ncomms 3612

31. E B, Giraldo NA, L L, et al. Estimating the population abundance of tissue-infiltrating immune and stromal cell populations using gene expression. Genome Biol. 2016;17(1):218. doi:10.1186/s13059-016$1070-5$

32. Aran D, Hu Z, Butte AJ. xCell: digitally portraying the tissue cellular heterogeneity landscape. Genome Biol. 2017;18(1):220. doi:10.1186/ s13059-017-1349-1

33. K M, M M. Potential of mesenchymal stem cells in anti-cancer therapies. Curr Stem Cell Res Ther. 2020.

34. Farhood B, Najafi M, Mortezaee K. CD8 + cytotoxic T lymphocytes in cancer immunotherapy: a review. $J$ Cell Physiol. 2019;234 (6):8509-8521. doi:10.1002/jcp.27782

35. G S, L F, D M, S L, Capron M. Involvement of eosinophils in the anti-tumor response. Cancer Immunol Immunother. 2012;61 (9):1527-1534. doi:10.1007/s00262-012-1288-3

36. Torphy RJ, Schulick RD, Yee Z. Newly emerging immune checkpoints: promises for future cancer therapy. Int J Mol Sci. 2017;18:12.

37. Su J, Ma Q, Long W, et al. LCTL is a prognostic biomarker and correlates with stromal and immune infiltration in gliomas. Front Oncol. 2019;9:1083. doi:10.3389/fonc.2019.01083

38. Fleurence J, Bahri M, Fougeray S, et al. Impairing temozolomide resistance driven by glioma stem-like cells with adjuvant immunotherapy targeting O-acetyl GD2 ganglioside. Int J Cancer. 2019.

39. Boussiotis VA, Charest A. Immunotherapies for malignant glioma. Oncogene. 2018;37(9):1121-1141. doi:10.1038/s41388-017-0024-Z

40. Belcaid Z, Phallen JA, Zeng J, et al. Focal radiation therapy combined with 4-1BB activation and CTLA-4 blockade yields long-term survival and a protective antigen-specific memory response in a murine glioma model. PLoS One. 2014;9(7):e101764. doi:10. 1371/journal.pone.0101764

41. Xue S, Hu M, Iyer V, Yu J. Blocking the PD-1/PD-L1 pathway in glioma: a potential new treatment strategy. J Hematol Oncol. 2017;10 (1):81. doi:10.1186/s13045-017-0455-6

42. Pitt JM, Vetizou M, Daillere R, et al. Resistance mechanisms to immune-checkpoint blockade in cancer: tumor-intrinsic and extrinsic factors. Immunity. 2016;44(6):1255-1269. doi:10.1016/j. immuni.2016.06.001

43. Chen YG, Satpathy AT, Chang HY. Gene regulation in the immune system by long noncoding RNAs. Nat Immunol. 2017;18(9):962-972. doi:10.1038/ni.3771

44. Chen F, Yin S, Zhu J, et al. IncRNA DGCR5 acts as a tumor suppressor in papillary thyroid carcinoma via sequestering miR-2861. Exp Ther Med. 2019;17(1):895-900. doi:10.3892/etm.20 18.7012
45. Wang YG, Liu J, Shi M, Chen FX. LncRNA DGCR5 represses the development of hepatocellular carcinoma by targeting the miR-346/ KLF14 axis. J Cell Physiol. 2018;234(1):572-580. doi:10.1002/ jcp. 26779

46. Dong HX, Wang R, Jin XY, Zeng J, Pan J. LncRNA DGCR5 promotes lung adenocarcinoma (LUAD) progression via inhibiting hsa-mir-22-3p. J Cell Physiol. 2018;233(5):4126-4136. doi:10.1002/ jcp. 26215

47. Tang T, Shan G, Zeng F. Knockdown of DGCR5 enhances the radiosensitivity of human laryngeal carcinoma cells via inducing miR-195. J Cell Physiol. 2019;234(8):12918-12925. doi:10.1002/ jcp. 27958

48. Huang J, Liu F, Liu Z, et al. Immune checkpoint in glioblastoma: promising and challenging. Front Pharmacol. 2017;8:242. doi:10. 3389/fphar.2017.00242

49. Ribas A, Wolchok JD. Cancer immunotherapy using checkpoint blockade. Science. 2018;359(6382):1350-1355.

50. Postow MA, Sidlow R, Hellmann MD. Immune-related adverse events associated with immune checkpoint blockade. $N$ Engl $J$ Med. 2018;378(2):158-168. doi:10.1056/NEJMra1703481

51. Reardon DA, Gokhale PC, Klein SR, et al. Glioblastoma eradication following immune checkpoint blockade in an orthotopic, immunocompetent model. Cancer Immunol Res. 2016;4(2):124-135. doi:10.1158/2326-6066.CIR-15-0151

52. Romani M, Pistillo MP, Carosio R, Morabito A, Banelli B. Immune checkpoints and innovative therapies in glioblastoma. Front Oncol. 2018;8:464. doi:10.3389/fonc.2018.00464

53. Wang X-L, Shi M, Xiang T, et al. Long noncoding RNA DGCR5 represses hepatocellular carcinoma progression by inactivating Wnt signaling pathway. J Cell Biochem. 2019;120(1):275-282.

54. T T, Shan G. DGCR5 promotes cancer stem cell-like properties of radioresistant laryngeal carcinoma cells by sponging miR-506 via Wnt pathway. J Cell Physiol. 2019;234(10):18423-18431. doi:10. $1002 /$ jcp. 28478

55. Galluzzi L, Spranger S, Fuchs E, López-Soto A. WNT signaling in cancer immunosurveillance. Trends Cell Biol. 2019;29(1):44-65. doi:10.1016/j.tcb.2018.08.005

56. S S, D D, Horton B, Gajewski TF. Tumor-residing Batf3 dendritic cells are required for effector $\mathrm{T}$ Cell trafficking and adoptive $\mathrm{T}$ cell therapy. Cancer Cell. 2017;31(5):711-723.e714. doi:10.1016/j. ccell.2017.04.003

57. S S, B R, Gajewski TF. Melanoma-intrinsic $\beta$-catenin signalling prevents anti-tumour immunity. Nature. 2015;523(7559):231-235. doi:10.1038/nature14404

58. Xue J, Yu X, Xue L, Ge X, Zhao W, P W. Intrinsic $\beta$-catenin signaling suppresses CD8 T-cell infiltration in colorectal cancer. Biomed Pharmacother. 2019;115:108921. doi:10.1016/j.biopha.2019. 108921

59. Castagnoli L, Cancila V, Cordoba-Romero SL, et al. WNT signaling modulates PD-L1 expression in the stem cell compartment of triple-negative breast cancer. Oncogene. 2019;38(21):4047-4060. doi:10.1038/s41388-019-0700-2

60. Li C-W, Lim S-O, Xia W, et al. Glycosylation and stabilization of programmed death ligand-1 suppresses T-cell activity. Nat Commun. 2016;7(1):12632. doi:10.1038/ncomms 12632

61. Shah KV, Chien AJ, Yee C, Moon RT. CTLA-4 is a direct target of Wnt/beta-catenin signaling and is expressed in human melanoma tumors. J Invest Dermatol. 2008;128(12):2870-2879. doi:10.1038/ jid. 2008.170 


\section{Publish your work in this journal}

OncoTargets and Therapy is an international, peer-reviewed, open access journal focusing on the pathological basis of all cancers, potential targets for therapy and treatment protocols employed to improve the management of cancer patients. The journal also focuses on the impact of management programs and new therapeutic agents and protocols on patient perspectives such as quality of life, adherence and satisfaction. The manuscript management system is completely online and includes a very quick and fair peer-review system, which is all easy to use. Visit http://www.dovepress.com/ testimonials.php to read real quotes from published authors.

Submit your manuscript here: https://www.dovepress.com/oncotargets-and-therapy-journal 